\title{
A Novel Indoor Wayfinding System Based on Passive RFID for Individuals with Cognitive Impairments
}

\author{
Yao-Jen Chang, Chien-Nien Chen \\ Department of Electronic Engineering \\ Chung Yuan Christian University \\ Chung-Li, Taiwan 320 \\ \{yjchang,jchen\}@cycu.edu.tw
}

\author{
Li-Der Chou \\ Dept. of Computer Science and \\ Information Engineering \\ National Central University \\ Chung-Li, Taiwan 320 \\ cld@csie.ncu.edu.tw
}

\author{
Tsen-Yung Wang \\ Institute of Health and Welfare Policy \\ National Yang Ming University \\ Taipei, Taiwan 112 \\ tywang@ym.edu.tw
}

\begin{abstract}
A novel wayfinding system is presented with an aim to increase workplace and life independence for cognitiveimpaired patients such as people with traumatic brain injury, cerebral palsy, mental retardation, schizophrenia, and Alzheimer's disease. It is based on passive RFID tags which embed the coordinate $(x, y, f l o o r)$. According to psychological model of spatial navigation and the requirements of rehabilitation professionals, PDAs prompting with spatial photos at the right time and place can assist cognitivelyimpaired persons with navigating indoors or on the road. To do so, passive RFID tags which can be imagined as a new traffic sign system, are posted to selected positions on routes. The navigational photos are served on demand to the user who uses the built-in RFID reader to sense the RFID tag when it is in eyesight range. A tracking function is integrated to timestamp the visited positions and issue alerts in case of anomalies. The tracking system increases the sense of security and also lowers the entry threshold to accepting the assistive technology. A prototype which consists of wayfinding devices and a tracking system, is designed and tested on a university campus. Compared to the sensor network approach, RFID tags are easier and faster to deploy and the cost is lower. The experimental results show the computer-human interface is friendly and the capabilities of wayfinding are reliable.
\end{abstract}

Keywords-Cognitive disability, ubiquitous computing, user interface, RFID, ontology.

\section{INTRODUCTION}

A growing literature [1] [2] [3] [4] [5] has begun to explore the process by which cognitive assistance, inside a smart home, is provided to occupants suffering from cognitive deficiencies such as Alzheimer's disease, traumatic brain injury, cerebral palsy, mental retardation, and schizophrenia, for the performance of their Activities of Daily Living (ADL). Putting on a larger scale, not only the home but also the community or even the city can be increased with intelligence to make people's lives easier, especially lives of those with less fortune. For example, the majority of otherwise-employable persons with mental impairments remain unemployed, rarely access appropriate community services, and are socially isolated. Wayfinding systems are an assistive technology targeting cognitive-impaired patients who are mobile and need to travel through both indoor and outdoor environments for work, shopping, socializing, therapy, and other purposes, thus increasing workplace and life independence.

In our study, RFID tags are used for personal wayfinding purposes. Passive RFID tags have no internal power supply, never run out of battery, and packaged in a rugged form, which all make them ideal for large scale deployment in the wayfinding infrastructure.

Keeping the users attitudes in mind, we propose a novel personal guidance system based on passive RFID tags for individuals with cognitive impairments. Such a personal guidance system will help them safely and effectively with personal wayfinding and, thus improving the quality of life without the great cost and inconvenience of special assistive services. Based on psychological models of spatial navigation, an individual carrying a handheld is instructed by a photo showing directions when she reaches a position on a planned trip. Every such photo is triggered by an RFID tag displayed at important positions, such as street intersections, exits, elevator doorways, and entrances to stairways. By sensing the RFID tag with his reader-ready handheld, the individual is able to receive guidance embedded within the photos just in time. Furthermore, to increase the sense of security and help with precautionary measures, a tracking interface is included in the system for authorized personnel to observe an individual's trajectory while the handheld device is being used. The tracking functions by recording the person ID, timestamping the visited position, elapsed time after leaving the last position, and expected arrival time to the next position. In case

Permission to make digital or hard copies of all or part of this work for personal or classroom use is granted without fee provided that copies are not made or distributed for profit or commercial advantage and that copies bear this notice and the full citation on the first page. To copy otherwise, to republish, to post on servers or to redistribute to lists, requires prior specific permission and/or a fee. PERVASIVEHEALTH 2008, 30 Jan - 1 Feb. Tampere, Finland

Copyright $\odot 2008$ ICST 978-963-9799-15-8

DOI 10.4108/ICST.PERVASIVEHEALTH2008.2506 
anomalies occur, such as individuals failing to reach a position en route or prolonged elapsed time between two adjacent positions, actions can be taken by the support team or family members to make sure things are all right.

The paper is organized as follows. In the next section, we survey the state of the art in the wayfinding research for individuals with cognitive impairments. Then, prototype design which draws upon psychological models of spatial navigation is presented. Implementations and results are shown followed by some concluding remarks.

\section{SURVEY}

Current methods in social services for aiding people with wayfinding are labor-intensive. For example, job coaches at several Taipei-based rehabilitation institutes, who work with individuals with mental impairments to support them in learning new jobs and maintaining paid employment, may work for weeks helping a person learn how to travel to and from work. Even then, the individual may at times still require assistance of one form or the other. While en route to the work, the person needs to be reminded by phones from the supporting group, or followed by the job coach invisible to the person, in order to keep things safe and in control. As a result the majority of otherwise-employable persons with cognitive impairments remain unemployed, rarely access appropriate community services, and are socially isolated [8] [9] [10].

Researchers at the University of Colorado have designed an architecture for delivering just-in-time transit directions to a PDA carried by bus users, using GPS and wireless technology installed on the buses [11]. The Assisted Cognition Project at the University of Washington has developed artificial intelligence models that learn a user behavior to assist the user who needs help [12]. Later a feasibility study [13] of user interface was carried by the same team, who found photos are a preferred medium type for giving directions to cognitively impaired persons in comparison with speech and text. However, they used a manual approach to decide when to send photos based on location information uploaded by the shadow support team. In addition, route personalization was not considered; only fixed routes can be planned ahead of the trips. Our research is one step forward beyond their pioneering work in [13].

\section{PROTOTYPE DESIGN}

The wayfinding system consists of two parts: a handheld PDA and a tracking system. The design draws upon the psychological models of spatial navigation, usability studies of interfaces by people with cognitive impairments, and the requirements based on interviews with nurses and job coaches at rehabilitation hospitals and institutes.
A. Wayfinding Handhelds

To relieve the job coaches from labor-intensive aids with wayfinding, a PDA is carried by the individual who has difficulty in indoor wayfinding or taking public transit to and from work. The PDA shows the just-in-time directions and instructions by displaying photos, triggered by passive RFID tags sensed by the PDA's built-in reader. See Figure 1 for the photo downloaded. However, the persons who carry the wayfinding PDAs may at times need assistance when they find themselves lost somewhere and cannot make themselves understood where they are. Note the system also works even for a stranger to this neighborhood.

\section{B. Placement of RFID}

The RFID tags are placed where patients may need to make decisions on which direction to take, such as a door or elevator. In many situations such as straight hall ways in indoor environments, no RFID tags need to be placed in the middle, since no changes of directions are expected to arise. Therefore, the number of RFID tags is limited to certain spots where they are actually needed instead of densely distributed everywhere.

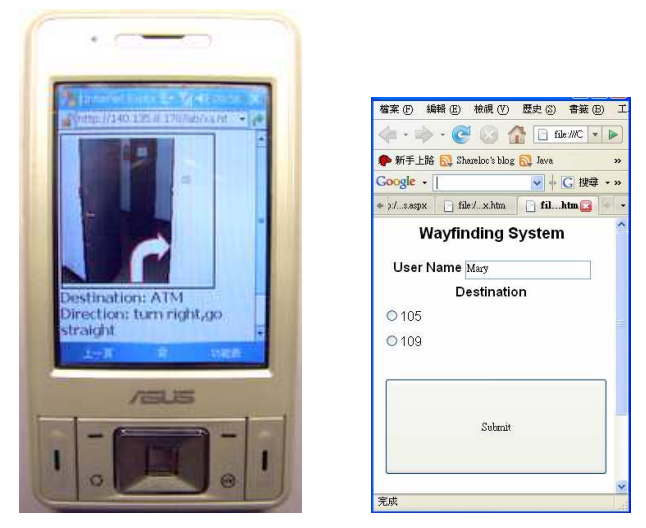

Figure 1: A just-in-time direction is shown on the wayfinding PDA

\section{IMPLEMENTATIONS AND EXPERIMENTAL RESULTS}

A prototype system is implemented for job coaches at several Taipei-based rehabilitation institutes funded by a government-sponsored social work program. The prototype consists of how PDA receives the photos, how RFID tags trigger downloading, what photos should be downloaded accordingly, and how individuals are tracked. The PDA is equipped with a screen size $320 * 240$, Wi-Fi 802.11g, Bluetooth, GPRS and an ISO 14443A RFID reader. The server is a Intel-based PC server for authenticating the users, planning a trip, serving photos upon requests from PDAs, and receiving timestamps for each position visited. The 
connectivity is provided via Wi-Fi on a campus with 410 Access Points. In places where $\mathrm{Wi}-\mathrm{Fi}$ is not available, GPRS can be used instead. The architecture is shown in Figure 2.

Experiments are designed to test the implemented prototype. Four routes in different combinations of stairways, elevators, and turns have been planned in the study. Route 1 involves no floor changes, while Route 2 is outdoors, Route 3 involves taking the stairs down one flight, and Route 4 involves using an elevator and taking the stairs down one flight. As shown in Figure 3, Route 4 is from Laboratory 517, which is located on the 5-th floor, to an ATM machine, which is at the entrance at the ground floor.

In order to accomplish the trip, positions on the route have to be passed successfully. All the positions are posted with the RFID tags, which are embedded with location information. Participants are shown the device and trained before the experiments. They are led to the starting location of each route and given the task of following the device's directions to a set destination. Participants are told their destination before starting their trips. After the user submits the choice being made, the wayfinding server writes a cookie to the client and maintains a session with this handheld.

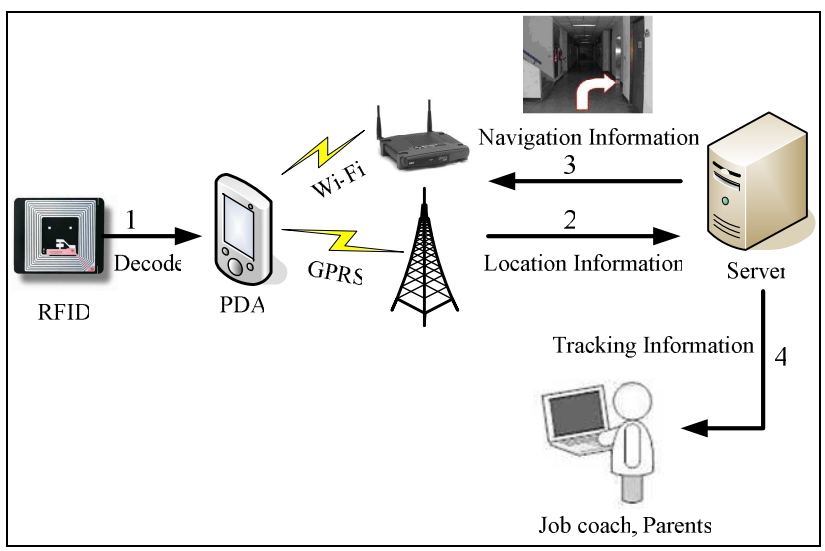

Figure 2: Architecture and system interaction of the wayfinding prototype. By sensing the RFID tag (1), the user PDA provides location information (2) sent over wi-fi, followed by the navigation server uses that location information to decide which photos to send (3). The user then follows the direction or prompt displayed on device. The navigation server records the positions, time, and user ID for the tracking purpose. A user interface is provided for job coaches or family members to retrieve the tracking information (4) which is then displayed on a map.

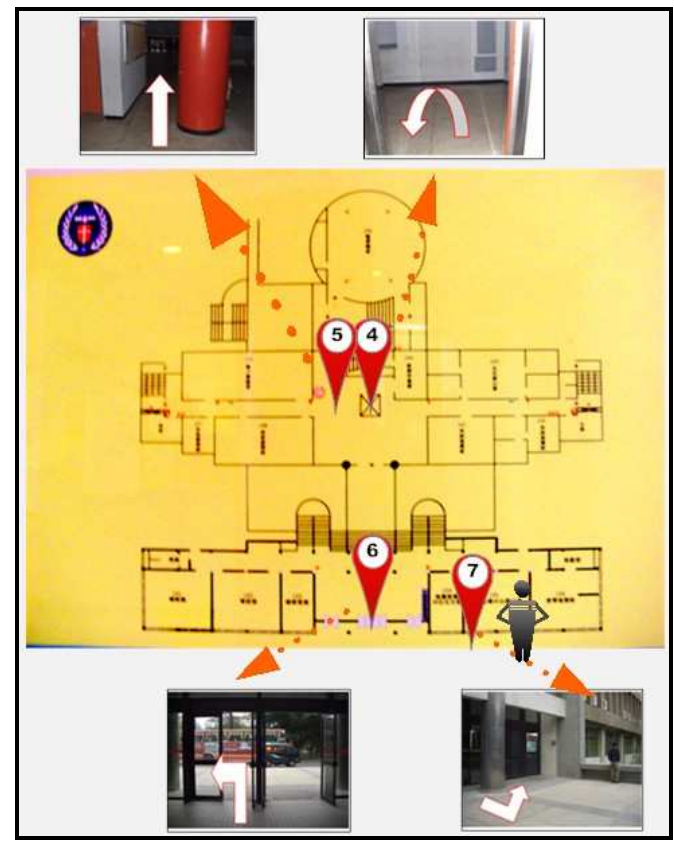

Figure 3: Floor plan of the ground level with an ATM machine as the destination of a trip.

In Table 1, we summarize the experimental outcomes based on the observations of the prototype design team. In the 96 trips made by 12 cognitively-impaired participants taking the four routes, twice on each route, respectively, the ratio of participants deviating from the set routes is $7.30 \%$. Note the ratio can depend on the extent to which participants suffer from mental disabilities, the complexity of routes, the degree of received training and self-practices, and the distractions the participants may encounter. We also do a participant survey regarding their subjective opinions on the device capability of navigation and quality of information provided by images, measured from 1 to 5 (poor to good). The overall impressions are satisfactory.

TABLE 1: Experiments of twelve cognitively-impaired participants taking the four routes, twice on each route

\begin{tabular}{|c|c|c|c|c|c|}
\hline $\begin{array}{l}\text { Partici- } \\
\text { pant }\end{array}$ & $\begin{array}{l}\text { Gen- } \\
\text { der } \\
/ \text { Lost }\end{array}$ & $\begin{array}{l}\text { Arrived } \\
\text { PDA } \\
\text { first time } \\
\text { (Yes/No) }\end{array}$ & $\begin{array}{l}\text { Capability } \\
\text { of } \\
\text { navigation } \\
(1 \sim 5, \text { poor } \\
\text { to good) }\end{array}$ & $\begin{array}{l}\text { Quality of } \\
\text { information } \\
\text { provided by } \\
\text { images } \\
(1 \sim 5)\end{array}$ \\
\hline 1 & F & $8 / 0$ & Y & 3 & 3 \\
\hline 2 & M & $7 / 1$ & Y & 3 & 3 \\
\hline 3 & M & $8 / 0$ & Y & 4 & 4 \\
\hline 4 & F & $7 / 1$ & Y & 4 & 4 \\
\hline 5 & M & $8 / 0$ & N & 3 & 5 \\
\hline 6 & F & $7 / 1$ & Y & 3 & 4 \\
\hline
\end{tabular}




$\left.\begin{array}{|c|c|c|c|c|c|}\hline 7 & \mathrm{M} & 7 / 1 & \mathrm{Y} & 2 & 4 \\ \hline 8 & \mathrm{M} & 7 / 1 & \mathrm{Y} & 4 & 4 \\ \hline 9 & \mathrm{M} & 7 / 1 & \mathrm{Y} & 4 & 4 \\ \hline 10 & \mathrm{~F} & 8 / 0 & \mathrm{Y} & 4 & 4 \\ \hline 11 & \mathrm{M} & 7 / 1 & \mathrm{Y} & 4 & 5 \\ \hline 12 & \mathrm{~F} & 8 / 0 & \mathrm{Y} & 4 & 4 \\ \text { Sciens }\end{array}\right]$ [1]

ACKNOWLEDGMENTS

The work presented in this paper has been funded by the National Science Council under grant numbers NSC 96-2627-E-008-001-.

\section{REFERENCES}

] Giroux S., Pigot H. "From Smart Homes to Smart Care, Assistive Technology Research Series. Proceeding of the 3rd International Conference on Smart homes and health Telematics," Proceedings of ICOST'05, Sherbrooke, Canada, 2005.

A primary security concern surrounding RFID technology is the illicit tracking of RFID tags. Tags which are worldreadable pose a risk to both personal location privacy and corporate/military security. In our study, the RFID tags are not carried by the individuals but fixed like road signs. Therefore, there are no security issues in terms of the tags. However, the tracking system is strongly protected by endto-end cryptographic communication protocols, such as SSL, and employing programming techniques of session control like session beans in Java, so that the privacy information does not leak to malicious persons.

There are some limitations to the PDAs and user interfaces. PDAs are fragile and not weather proof. Therefore, protective measures need to be taken to keep them in good maintenance from frequent use. A challenge to us in the experiments is that strong sunshines can make the screen hardly viewable no matter what is downloaded and shown. Fortunately, the same problem seldom arises in indoor wayfinding.

\section{CONCLUSIONS}

In this paper, we present a wayfinding prototype system based passive RFID tags for individuals with cognitive impairments. The design draws upon the cognitive models of spatial navigation and consists of wayfinding devices and a tracking system. Compared to the sensor network approach, it is easy to deploy because of low cost and short time frame. The prototype is implemented and tested with routes in the campus. The results show the prototype is user friendly and promising with high reliability. The success ratio can depend on the extent to which participants suffer from mental disabilities, the complexity of routes, the degree of received training and self-practices, and the distractions the participants may encounter. Limitations to PDAs and user interfaces are discussed and then challenges are identified. A vision to the future is to realize a new system of traffic signs coexisting with the current one for the betterment of cognitive-impaired individuals with increased life independence.
[2] Bauchet J., Mayers A. "Modelisation of ADL in its Environment for Cognitive Assistance," Proc. of the 3rd International Conference on Smart homes and health Telematic, ICOST'05, Sherbrooke, Canada, pp. 3-10. 2005.

[3] Boger J., Poupart P., Hoey J., Boutilier C., Fernie G. and Mihailidis A."A Decision-Theoretic Approach to Task Assistance for Persons with Dementia." Proc. of the International Joint Conference on Artificial Intelligence (IJCAI'05), pp. 1293-1299, Edinburgh, Scotland, 2005.

[4] Fatiha L., Lefebvre B."A cognitive system for a smart home dedicated to people in loss of autonomy," Proc. of the 3rd Int. Conference on Smart homes and health Telematic, ICOST'05, Sherbrooke, Canada, pp. 245-254. 2005.

[5] Pigot H., Mayers A., Giroux S."The intelligent habitat and everyday life activity support," Proc. of the 5th International conference on Simulations in Biomedicine, April 2-4, Slovenia, pp. 507-516. 2003.

[6] Rogers, E. M., Diffusion of innovations (4th ed.), New York: The Free Press, 1995.

[7] Kintsch, A. \& dePaula, R., "A Framework for the Adoption of Assistive Technology," Proceedings of SWAAAC 2002: Supporting Learning Through Assistive Technology, pp. E3 1-10, 2002.

[8] Bond, G. R. and R. P. Liberman. Vocational rehabilitation. Handbook of psychiatric rehabilitation. New York, Macmillan. 1992.

[9] Bond, G. R., R. E. Drake, et al. "Supported employment for people with severe mental illness: A review." Psychiatric Services. 1996.

[10] Goodwin, S. Comparative mental health policy: From institutional to community care. Thousand Oaks: Sage. 1997.

[11] S. Carmien, M. Dawe, G. Fischer, A. Gorman, A. Kintsch, and J. F. Sullivan. "Socio-technical environments supporting people with cognitive disabilities using public transportation." ACM Trans on Computer-Human Interaction, 12(2):233 - 262, Jun 2005.

[12] L. Liao, D. Fox, and H. Kautz. "Learning and inferring transportation routines." In Proc of the 19th Natl Conf on AI, 2004.

[13] L. Liu, H. Hile, H. Kautz, G. Borriello, P.A. Brown, M. Harniss, K. Johnson, "Indoor Wayfinding: Developing a Functional Interface for Individuals with Cognitive Impairments," Proceedings of Computers \& Accessibility, ASSETS 2006, pp.95 - 102, October 2006.

[14] Paul Bausch, Matthew Haughey, and Meg Hourihan. We Blog: Publishing Online with Weblogs. John Wiley \& Sons, New York, Chapter 8, 2002. 